Hans Ziegenthaler · Lutz Brückner

Orthopädische Abteilung der Moritz-Klinik GmbH, Fachklinik für Neurologie und Orthopädie AHB-Klinik, Bad Klosterlausnitz

\title{
Mitteldeutsches Rehabilitationsdreieck
}

\section{Konzept zur Koordination der Rehabilitation Brandverletzter}

\section{Zusammenfassung}

Mit dem Fortschritt im Rettungswesen, in der Akutmedizin und in der plastischen Chirurgie steigen die Überlebenschancen für Brandverletzte. Die physisch und zumeist auch psychisch traumatisierten Patienten bedürfen einer intensiven Weiterbehandlung. Ein individuell abgestimmtes Nachsorgeprogramm mit speziellen Anforderungen an die medizinisch-funktionelle Behandlung, die umfassende soziale und berufliche Reintegration und im Besonderen an die psychologische Betreuung ist erforderlich. Anhand eigener Erfahrungen wird ein interdisziplinär getragenes Konzept zur Brandverletztenrehabilitation vorgestellt.

\section{Schlüsselwörter}

Verbrennung $\cdot$ Rehabilitation - Narben . Fallmanagement $\cdot$ Soziale Reintegration urch ein gut organisiertes Rettungssystem, Fortschritte in der Intensivmedizin sowie innovative Transplantationsund Hautersatzverfahren überleben in den Brandverletztenzentren heute etwa 4/5 aller Brandverletzten [1]. Darüber hinaus können durch den frühen Einsatz plastisch-rekonstruktiver Verfahren nicht selten Amputationen abgewendet und funktionelle Fähigkeiten erhalten werden.

Dennoch weisen Brandverletzte nach der Phase der Akutbehandlung häufig weit reichende Einschränkungen an körperlicher Unversehrtheit, an funktionellen Fähigkeiten und an sozialer Integrität auf. Hier finden sich Ansatzpunkte, die zugleich Herausforderung und Chance für einen gezielten und qualifizierten, sich in Phasen gestaltenden Prozess medizinischer, sozialer und beruflicher Rehabilitation darstellen. Dessen Ziel ist in der Verbesserung funktioneller und sozialer Fähigkeiten zu sehen, was eine Rückkehr in ein Leben, das von Selbstständigkeit und wiedererlangter Souveränität geprägt ist, ermöglicht.

Mit dem Aufbau der Brandverletztenzentren (BVZ) in der BG-Klinik "Bergmannstrost“ Halle, Saale und am Städtischen Klinikum "St. Georg“ in Leipzig entstand zwangsläufig ein Rehabilitationsbedarf für Brandverletzte vorwiegend aus den Bundesländern Sachsen, Sachsen-Anhalt und Thüringen. Durch gezielte Hospitationen aller Berufsgruppen und Weiterbildungen auf dem Gebiet der Verbrennungsmedizin entstand in Zusammenarbeit mit den genannten BVZ ein einheitliches Rehabilitationskonzept.

\section{Epidemiologische Daten}

Von Juni 1997-Mai 2001 wurden in der Moritz-Klinik 58 Patienten nach schwerem Verbrennungstrauma rehabilitiert. Als führende Kostenträger waren dabei die Berufsgenossenschaften $\mathrm{zu}$ verzeichnen (Abb. 1). Daher wurde mit dem Landesverband der gewerblichen $\mathrm{Be}$ rufsgenossenschaften Hessen-Mittelrhein-Thüringen im Juni 2000 eine Sondervereinbarung für Brandverletzte in der stationären Rehabilitation, die die speziellen Anforderungen an materiellen und personellen Ressourcen der Rehabilitationsklinik berücksichtigt, abgeschlossen.

In Tabelle 1 sind epidemiologische Daten zu den Brandverletzten im oben genannten Zeitraum aufgeführt. Anhand der Übersicht zur Verweildauer

\footnotetext{
(c) Springer-Verlag 2003

Dr. Hans Ziegenthaler Orthopädische Abteilung der Moritz-Klinik GmbH, Fachklinik für Neurologie und Orthopädie AHB-Klinik, Hermann-Sachse-Straße 46, 07639 Bad Klosterlausnitz, E-Mail:mok@ugos.de, Tel.: 036601-49461, Fax: 036601-49960
} 


\section{H.Ziegenthaler · L. Brückner}

\section{Rehabilitation \\ in central Germany. \\ Strategy for coordination \\ of rehabilitation in burn patients}

\section{Abstract}

The chances of survival after burn injury have increased in recent times with the advances in rescue services and emergency medicine and the innovations in surgical treatment. At present, more than $80 \%$ of severe burn-injured patients probably survive. After special treatment in a burns center, victims need treatment in an inpatient rehabilitation care center with special equipment. This article outlines postburn rehabilitation, illustrated with reference to the example of our clinic. It also describes specific interventions directed at medical and social rehabilitation.

\section{Keywords}

Burns · Rehabilitation - Scar formation · Case management $\cdot$ Social reintegration

\section{Abb. 1 Kostenträger der BV-Rehabilitation vom Juni 1997- Mai 2001 in der Moritz-Klinik}

wird deutlich, dass etwa 1/4 der Brandverletzten bereits von einer Behandlungszeit bis zu 28 Tagen profitierte und in die ambulante Betreuung entlassen werden konnte. Die mittlere Verweildauer lag bei 43,2 Tagen. In einem Teil der Fälle waren allerdings lange und intensive Nachbehandlungszeiträume notwendig. In dieser Gruppe subsumierten sich auch Brandverletzte, die primär auf einer Station der Frührehabilitation aufgenommen wurden. Dort können die Möglichkeiten und Vorteile der komplexen stationären Rehabilitation mit dem noch vorhandenen erhöhten Bedarf an pflegerischen Aufwendungen, z. B. bei der Versorgung von Trachealkanülen oder der parenteralen Ernährung, abgesichert werden. In einem solchen Fall ergänzen sich Frührehabilitation und spätere Anschlussbehandlung.

Entgegen der Annahme ließ sich statistisch keine Korrelation zwischen der Verweildauer und dem Alter bzw. der betroffenen Körperoberfläche nachweisen. Obwohl die untersuchte Gruppe relativ klein war, sehen wir darin eine Bestätigung, dass in der Rehabilitation Brandverletzter der Grad der individuellen Betroffenheit den Maßstab in der Bewertung der Rehabilitationsbedürftigkeit bestimmt.

\section{Braucht es Zentren zur Rehabilitation von Brandverletzten?}

Brandverletztenzentren sind prinzipiell für Aufgaben in der Akutversorgung konzipiert. Nur in wenigen Zentren wird darüber hinaus die weiterführende Rehabilitationsbehandlung sichergestellt.

Im ambulanten Bereich und in nicht spezialisierten Rehabilitationskliniken mit geringen Fallzahlen ( $<20 / J a h r)$ kann die spezifische komplexe Nachbehandlung für Brandverletzte nicht sicherge-

\begin{tabular}{ll} 
Tabelle 1 & \\
Epidemiologische Daten zur BV-Rehabilitation & \\
Daten & Werte \\
\hline Zeitraum & Juni 1997-Mai 2001 \\
Brandverletzte & 58 \\
Geschlecht & \\
$\quad$ Männlich & 40 \\
$\quad$ Weiblich & 18 \\
Alter & \\
$\quad$ Altersdurchschnitt [Jahre] & 42,1 \\
$\quad$ Spannweite (Minimum-Maximum) [Jahre] & $10-73$ \\
Rehabilitationszeitraum & \\
Im Mittel [Tage] & 43,2 \\
Spanne (Minimum-Maximum) [Tage] & $18-105$ \\
$\quad<4$ Wochen & $23,2 \%$ der Brandverletzten \\
$\quad 4-7$ Wochen & $42,8 \%$ der Brandverletzten \\
$\quad>8$ Wochen & $34,0 \%$ der Brandverletzten \\
Betroffene Körperoberfläche (Grad Ilb und III) & \\
$\quad$ Mittel [\%] & $31,7 \%$ \\
Spannweite [\%] & $5 \%$ (Gesicht/Hals)-70\% \\
&
\end{tabular}




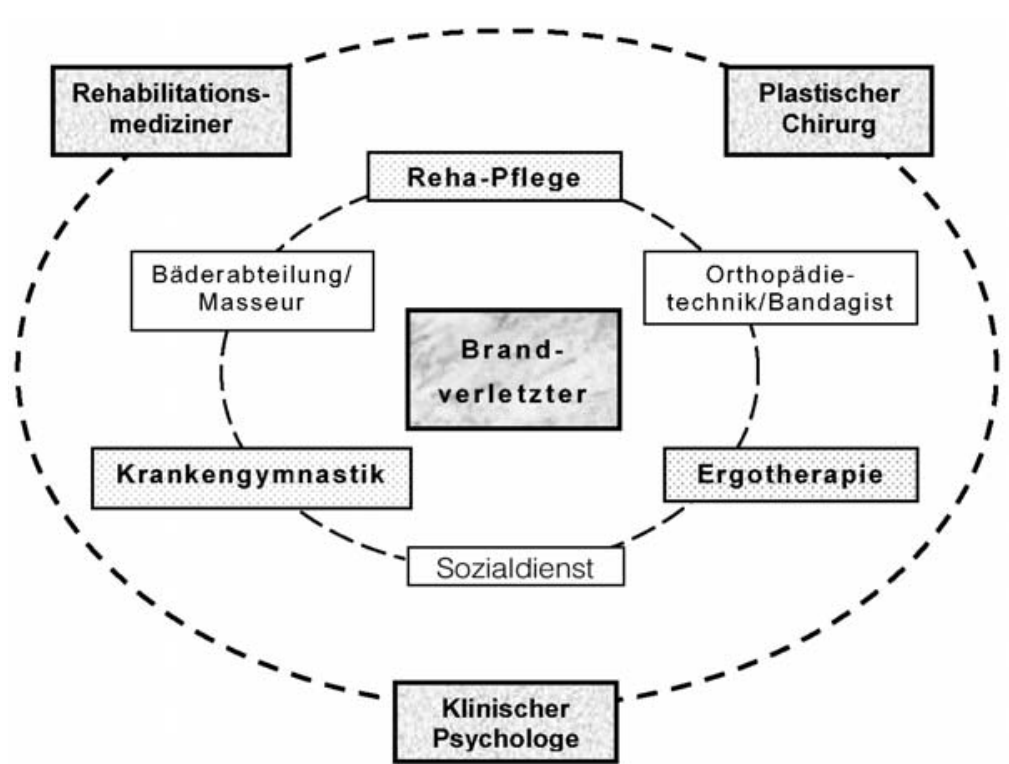

Abb. $2 \Delta$ Elemente des multiprofessionellen, interdisziplinären Rehabilitationsteams

stellt werden. Daher fordert der Hauptverband der gewerblichen Berufsgenossenschaften, die Rehabilitation Brandverletzter in einem Schwerpunktzentrum durchzuführen [4]. Hier werden mit hoher Struktur- und Prozessqualität die erforderlichen medizinisch-funktionellen Behandlungen, eine besonders differenzierte psychologische Betreuung und die Möglichkeiten zur umfassenden sozialen und beruflichen Reintegration garantiert. Neben der gezielten Koordination des Rehabilitationsteams wird durch ein individuelles fallbezogenes Rehabilitationsmanagement der effektive Einsatz personeller und apparativer Ressourcen sichergestellt.

\section{Rehabilitationsprozess}

In der Behandlung Brandverletzter zeichnet sich sowohl im Akut- als auch im Rehabilitationsbereich ein phasenhafter Verlauf ab. Dementsprechend werden befundorientierte Zielsetzungen im Team definiert und ergebnisorientierte Therapiemaßnahmen ausgewählt.

Mit der Übernahme des Brandverletzten in ein Rehabilitationszentrum vollzieht sich ein wesentlicher Schritt hin zum Übergang ins alltägliche und zugleich in ein neues Leben. Die Phase des Ringens um das Überleben, des Umsorgtseins, aber auch der Betriebsamkeit auf der Intensiveinheit ist überwunden. Nun stehen die Verbesserung der posttraumatischen Lebensqualität, das Wie- dererlangen von Selbstständigkeit und Eigenverantwortung - aber auch die Auseinandersetzung mit dem „Anderssein" - im Vordergrund. In wöchentlichen Teamkonferenzen werden mit Ausrichtung auf Fernziele unter Leitung des erfahrenen Rehabilitationsmediziners die Zielsetzungen anhand der aktuellen Befunde koordiniert. Durch enge Kontakte zu den im BVZ tätigen Chirurgen können plastisch-rekonstruktive Eingriffe und deren Zeitpunkt diskutiert und so das Timing operativer und konservativer Maßnahmen optimiert werden. Dies erfordert flexible Rehabilitations- und Verwaltungsstrukturen, die den ggf. sequenziellen Einsatz der verschiedenen Module, von der Frührehabilitation bis zur teilstationären oder gezielten ambulanten Nachsorge, im Einzelfall sicherzustellen vermögen.

\section{Elemente des \\ Rehabilitationsprozesses und des Rehabilitationsteams}

Mit der Erstellung eines Stufenmanagements für die medizinische, soziale und berufliche Rehabilitation sowie die psychologische Betreuung werden Therapeuten aus den verschiedensten Berufsgruppen in den Rehabilitationsprozess eingebunden (Abb. 2). Unser Rehabilitationskonzept ist komplex und vielseitig. Nur so kann es den individuellen Ansprüchen des Brandverletzten gerecht werden. Erst die klare Strukturie- rung des Tagestherapieplans garantiert die Umsetzung der notwendigen Behandlungen. Bei Gleichstellung aller Mitglieder im Therapieensemble sind es neben der rehabilitativen Krankenpflege die Narbenbehandlung, die Krankengymnastik sowie die Ergotherapie, die sich durch die Intensität der Behandlungen hervorheben.

\section{Rehabilitative Krankenpflege}

Die Betreuung Schwerbrandverletzter erfordert eine außerordentliche pflegerische Kompetenz. Zeitlicher Umfang und Intensität der Krankenpflege übersteigen täglich das gewöhnliche Maß. Ein wesentlicher Bestandteil sind die Pflege der Haut und die Versorgung noch bestehender Wunden. Per Definition sind dies keine Aufgaben der rehabilitativen Krankenpflege. Rezidivierende, meist oberflächliche Wunden und Epitheldefekte durch Spannungsblasen sind jedoch für Brandverletzte typisch. Somit entsteht Bedarf nach qualifizierter Weiterbildung und intensiver Auseinandersetzung mit modernen Konzepten des Wundmanagements. Im Stationsalltag und beim Training der Aktivitäten des täglichen Lebens (ADL) am Morgen, mit der Haut- und Wundpflege nach der Narbenbehandlung sowie der

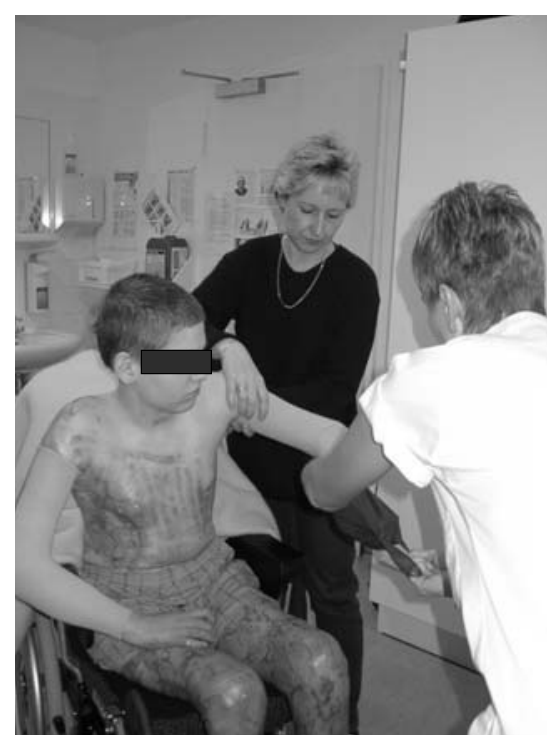

Abb. $3 \Delta$ Beim Anlegen der Kompressionsbekleidung bedarf es gerade am Anfang umfangreicher Hilfe. Dabei wird der Brandverletzte zur Selbsthilfe angehalten und ggf. Angehörige im Handling und im Umgang mit Hilfsmitteln geschult 
Haut-, Wund- und Körperpflege am Abend bietet sich für die Pflegekraft immer wieder die Möglichkeit für begleitende Gespräche. Dabei wird neben der fachlichen Anleitung die Notwendigkeit zur Motivation für Selbsthilfe genutzt. Über die Selbstpflege wird der Selbsthilfegedanke aktiviert. Hinzukommt die Verantwortung bei der psychologischen Unterstützung und Begleitung des Brandverletzten im gesamten Rehabilitationsprozess, mit der daraus resultierenden emotionalen Nähe (Abb. 3).

\section{Spezifische Narbenbehandlung}

Die spezifische Narbenbehandlung (Abb. 4) ist integraler Bestandteil der Behandlung und dauert täglich (Montag bis Samstag) bis zu $1 \mathrm{~h}$. Ein medizinisches Bad mit Betaisodona ${ }^{\circledR}$, Kamille oder Weizenkleie als Zusatz dient der Vorbereitung. Je nach dem Ausmaß der betroffenen Körperoberfläche arbeiten 1-2 Therapeuten an einem Patienten. Die Narbenmassage enthält Elemente der Lymph- und Bindegewebsmassage $[5,7]$. Mit Grifftechniken aus der manuellen Lymphdränage und durch die Stimulation zentraler Lymphknoten erfolgt die Anregung zentraler Abflusswege. Als Koppelmedium dient eine spezielle fetthaltige Narbenpflegesalbe (Unguentum cordes $\left.{ }^{\circledR}\right)$. Weiter wird dann

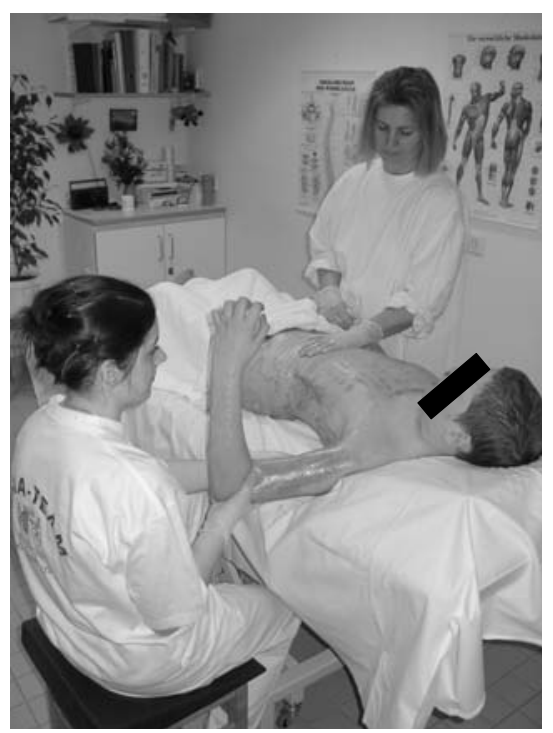

Abb. $4 \Delta$ Je nach Ausdehnung und Lokalisation der Verbrennung, hier $80 \%$, erfolgt die spezielle Narbenmassage durch 1-2 Therapeuten über 60 min täglich von Montag bis Samstag
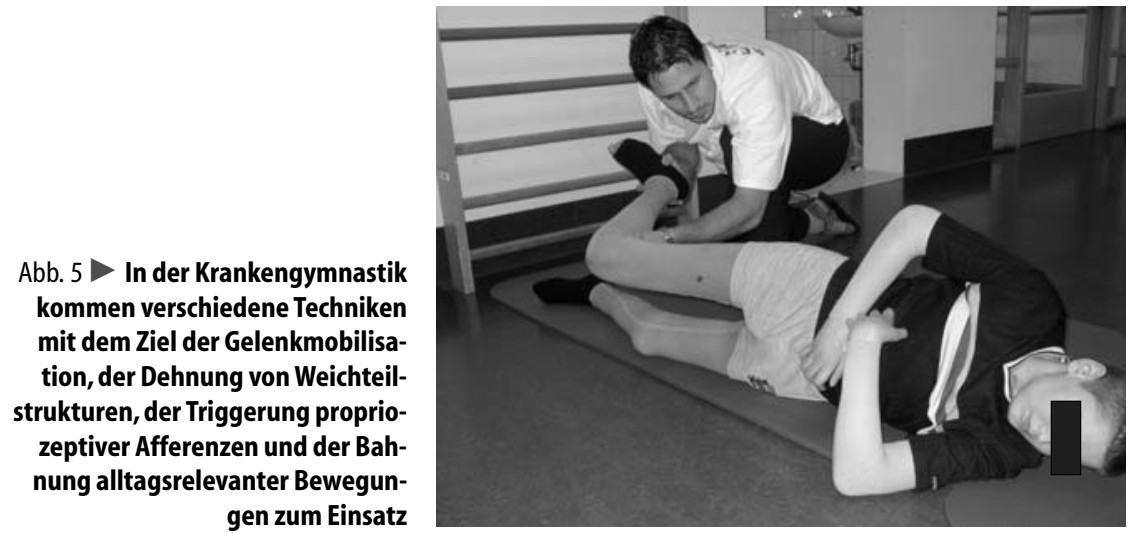

lymphbahntypisch, aber mit relativ starken Tiefenfriktionen gearbeitet. In den Bindegewebsschichten wird so die Narbe von kontraktilen Elementen und anderen Weichteilen gelöst. Der lokal entstauende Effekt verbessert die trophische Situation. Zugleich wird durch vegetative Reize und in Kombination mit der Kompressionsbekleidung ein positiver Effekt auf bindegewebige Strukturen erwartet. Neben dem Zuwachs an Geschmeidigkeit resultiert eine Verbesserung der Beweglichkeit betroffener Gelenke.

\section{Kompressionsbekleidung}

Im Reifungsprozess der Narben, der sich über 10-24 Monate erstrecken kann, wirkt kontinuierlicher Druck der hypertrophen Narbenbildung entgegen. Optimal sitzende Kompressionsbekleidung (Abb. 3) übt einen konstanten Druck (etwa $15-40 \mathrm{mmHg}$ ) auf die Haut aus. In besonders konturierten Problemzonen (Achsel, Ellenbeuge, Handfläche, Gesicht) kann er über Einlagematerialien aus Silikon erreicht werden. Silikone selbst, als Gel oder Platte verwendet, haben auch ohne Kompression einen positiven Einfluss auf die Narbenentwicklung. Der eigentliche Wirkmechanismus ist dabei noch ungeklärt [3].

Erst das regelmäßige Tragen und v. a. der faltenfreie Sitz gewährleisteten den gewünschten Effekt der besseren Narbenreifung. Die Kompression ist aber über den gesamten Tag - $24 \mathrm{~h}-$ notwendig. Muskelzuwachs und Rückgang von Schwellungen verändern die Passform gerade in den ersten Monaten, sodass regelmäßige Kontrollen der Passform und ggf. Anpassung (z. B. über Ab- näher) oder Neuverordnung und -anfertigung notwendig sind.

\section{Krankengymnastik}

In Abhängigkeit vom Ausmaß der Brandverletzung und der Dauer der Akutbehandlung bestehen eine oft schwere allgemeine Dekonditionierung, muskuläre Schwäche sowie Störungen vegetativer Funktionen. Verschiedene Regulationstherapien zur Optimierung einzelner Organfunktionen sowie Möglichkeiten für ein Koordinations- und Ausdauertraining kommen dann zur Anwendung.

In der Bewegungstherapie kommen

1. Techniken der klassischen Krankengymnastik,

2. Techniken der manuellen Medizin, 3. Techniken auf neurophysiologischer Grundlage

u. a. mit dem Ziel der

- Gelenkmobilisation,

- Dehnung von Weichteilstrukturen,

- Triggerung propriozeptiver Afferenzen und der Anbahnung alltagsrelevanter Funktionen zum Einsatz (Abb. 5).

Oftmals gilt es primär an der ,aufrechten Haltung" (Geradehalten des Kopfs, Strecken von Armen und Beinen) und dem Gefühl für die „Mitte des Körpers“ $\mathrm{zu}$ arbeiten.

Von Bewegungstherapie im Einzelbewegungsbad profitiert der Brandverletzte. Dieses muss gesonderten hygienischen Bedingungen genügen. Im Vergleich zu einer ausschließlichen Chlor- 


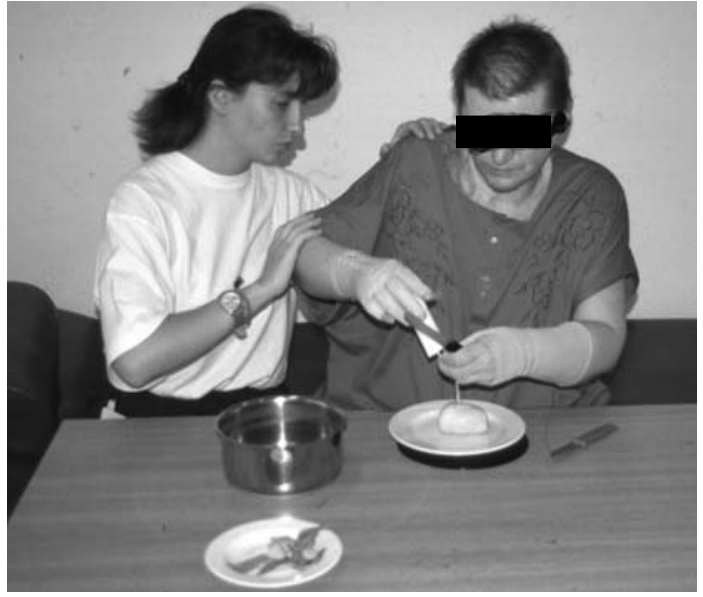

Abb. $6<$ Funktionelles Training unter alltagsrelevanten Bedingungen. Nutzung adaptierter Hilfsmittel im Haushaltstraining mit angelegter Kompressionsbekleidung (Gabel mit Griffverdickung). Die Ergotherapeutin korrigiert Ausweichbewegungen

desinfektion scheint eine Kombination mit einer Ozondesinfektion besser geeignet. Ungünstige Hautreaktionen konnten dabei nicht festgestellt werden. Durch die allgemein gute Akzeptanz von Wassertherapie wird beim Brandverletzten die Scheu, sich öffentlich mit dem veränderten Äußeren zu zeigen, schrittweise abgebaut. Dieser Aspekt sollte in die Therapie mit einbezogen werden.

\section{Ergotherapie und Aktivitäten des täglichen Lebens}

Ergotherapie schafft grundlegende Voraussetzungen für das Heranführen des Brandverletzten an den Alltag (Abb. 6) bis hin zum Wiedererlernen beruflicher und hobbytechnischer Fähigkeiten unter folgenden Zielsetzungen:

- Abbau von Ödemen, Funktionseinschränkungen, Fehlstellungen und Kontrakturen

- Förderung der Narbenreifung sowie Begrenzung durch Narben bedingter Bewegungseinschränkungen und Kontrakturen

- Erreichen des bestmöglichen Bewegungsumfangs, der normalen Muskelkraft und Sensibilität

- Wiedereingliederung in das soziale Umfeld (familiär und beruflich)

Neben aktiven und passiven Bewegungsübungen sowie dem Aufbau der Muskelkraft ist das Dehnen der Narbenzüge und kontrakter Strukturen aus funktioneller Sicht ebenso wichtig wie die Lagerung und Schienenbehandlung und die Sensibilitätsschulung sowie die Versorgung und das Training mit not- wendigen Hilfsmitteln. Funktionseinschränkungen sind letztlich so gering wie möglich zu halten [6].

\section{Psychologische Aspekte}

Mit der bleibenden Verletzung der menschlichen Haut ist es auch zur Verletzung einer besonderen Grenzzone, die sowohl das Gefühl der Unversehrtheit als auch der persönlichen Integrität und zugleich die Quelle sensorischer Erfahrung in der Entwicklung des Körperschemas repräsentiert, gekommen. Daher ist die psychologische Betreuung in der Rehabilitation Bandverletzter ein äußerst wichtiger Faktor. Zu lernen, sich mit dem neuen Äußeren, mit sich selbst und den neuen Lebensumständen auseinanderzusetzen, sich also so zu akzeptieren, wie man ist - so wie man aussieht -, ist ein Weg, auf dem Begleitung notwendig ist. Das Brandereignis selbst, aber auch die Phase der Intensivbetreuung können zu einer seelischen Traumatisierung beitragen. Psychische Auffälligkeiten wie z. B. Angstzustände, Scham- und Schuldgefühle in unterschiedlicher Ausprägung, eine gestörter Krankheitsverarbeitung, aber auch ausgeprägte posttraumatischen Belastungsreaktionen (PTSD) sind häufig und werden in der Literatur bei $30-50 \%$ der Brandverletzten angegeben [2]. Diese Probleme frühzeitig zu erkennen und einer geeigneten Intervention zuzuführen, erscheint bedeutsam. In der klinischen Psychologie werden zudem neuropsychologische Defizite differenziert und einer geeigneten Therapie zugeleitet. Nicht selten wird deren Einfluss auf die Krankheitsverarbeitung bei den psychisch traumatisierten Brandverletzten übersehen. Differenzialdiagnostisch sind prädisponierende Faktoren von irreversiblen hypoxischen und toxischen hirnorganischen Veränderungen abzugrenzen. Spezialkenntnisse und klinische Erfahrung in der Psychologie traumatisierter Opfer, in der Psychiatrie und Neuropsychologie sind unabdingbar.

\section{Begleitverletzungen und Amputationen}

Das Verbrennungstrauma begleitende Schäden innerer Organe, neurologische Komplikationen oder Probleme, die sich aus einem Inhalationstrauma ergeben, erfordern die ständige Präsenz eines Pools an Spezialisten im Rehabilitationszentrum. Kenntnisse im Umgang mit Trachealkanülen sind obligat. Verletzungen an Sinnes- oder Urogenitalorganen verlangen unproblematische Konsultationsmöglichkeiten bei Fachärzten. Bei einem methicillinresistenten Keimspektrum (MRSA) muss die Rehabilitation wie im Akuthaus unter einem besonderen Hygieneregime realisiert werden.

Amputationen bei Brandverletzten stellen eine zusätzliche Herausforderung dar. Probleme ergeben sich häufig bei der Belastungsfähigkeit der Stümpfe. Bevor ein vernarbter oder mit Transplantaten gedeckter Stumpf eine Prothese funktionsfähig trägt, vergeht in Abhängigkeit von der Narbenreifung oft eine lange Zeit. Daher stehen am Anfang stumpfabhärtende Maßnahmen, Übungen zum Gleichgewicht, zur Funktionsanbahnung sowie eine konsequente Kompressionstherapie. Eine $\mathrm{zu}$ frühe, auf noch nicht belastungsfähige Haut treffende Prothesenversorgung erhöht die Gefahr von Hautirritationen und somit von Stumpfinfektionen. Durch die Schrumpfung der Narbe und die Weichteilveränderungen wäre eine ständige Nachbesserung der Stumpfbettung erforderlich. Die „beste“ die, die den zu erwartenden Funktionsbedarf bzw. Aktivitätsgrad, der individuell sehr verschieden sein kann, am besten gerecht wird. Zur Beurteilung bedarf es neben der entsprechenden Fachund Sachkenntnis einer ausreichenden prothetischen Erfahrung. 


\section{Soziale Wiedereingliederung}

Eine frühzeitige soziale Reintegration in das Alltags- und Berufsleben wird für den Betroffenen durch Beratung im Sozialdienst, die Vermittlung von Kontakten zum Reha- oder Berufsberater gefördert. Arbeits- und Belastungserprobungen sind durch die Kooperation mit einem Berufsförderungswerk möglich. Angehörige werden in den Rehabilitationsprozess mit eingebunden, über die Gesundheits- und Folgeschäden sowie weitere Maßnahmen zu Hause informiert. Durch ihre Teilnahme an einzelnen Therapien können auch ihnen entsprechende Übungen, Lagerungstherapien und insbesondere der Umgang mit der Kompressionsbekleidung und Schienen vermittelt werden.

Bei der Entlassung werden schriftliche Hinweise für die Kompressionsbekleidung (Tragedauer, Handhabung, Pflege), für Selbstübungen sowie zur Hautpflege ausgehändigt. Sie beinhalten ebenso Kontaktadresse und -telefon zum Brandverletztenzentrum, zur Rehabilitationseinrichtung und $\mathrm{zu}$ den Selbsthilfegruppen. Ein Termin zur Wiedervorstellung im Brandverletztenzentrum wird vereinbart.

\section{Resümee}

Aus der Zusammenarbeit mit den Brandverletztenzentren in Halle und in Leipzig hat sich ein Rehabilitationsverbund entwickelt, der den individuellen Besonderheiten des Brandverletzten mit seinen weitreichenden Einschränkungen an körperlicher Unversehrtheit, funktionellen Fähigkeiten und sozialer Integrität gerecht wird und die Schnittstellenproblematik auf ein Minimum reduziert. Das 2000 weiterentwickelte Rehabilitationskonzept hat sich ebenso wie gegenseitige Hospitationen und gemeinsam gestaltete Weiterbildungsveranstaltungen bewährt.

Mit der Abstimmung des Übernahmezeitpunkts, der Behandlungsschwerpunkte und sekundär notwendiger plastisch-chirurgischer Eingriffe erfährt der Rehabilitationsprozess quantitativ und qualitativ eine Optimierung.

Die aus dem Fortschritt im Akutbereich resultierenden einzigartigen Chancen für die medizinische und soziale Rehabilitation Brandverletzter fordern neben der Struktur- und Prozessqualität in spezialisierten Rehabilitationszentren die Möglichkeiten zur flexiblen Führung des Rehabilitationsprozesses, um den gestellten Erwartungen gerecht zu werden.

\section{Literatur}

1. Deutschsprachige Arbeitsgemeinschaft für Verbrennungsbehandlung (2001) Statistik 2000 zur Jahrestagung der Deutschsprachigen Arbeitsgemeinschaft für Verbrennungsbehandlung (DAV), 7.-10.1.2001, Obertauern, Österreich

2. Faber AW, Klasen HJ, Sauer EW, Vuister FM (1987) Psychological and social problems in burn patients after discharge - A follow-up study. Scand J Plast Reconstr Surg 21: 307-309

3. Fulton FE (1995) Silicone gel sheeting for the prevention and management of evolving hypertrophic and keloid scars. Dermatol Surg 21: 947-951

4. Germann G, Wentzensen A, Brandt KA, Steinau HU (1999) Denkschrift für die Rehabilitation Brandverletzter - Empfehlung des Hauptverbandes der gewerblichen Berufsgenossenschaften. Hauptverband der gewerblichen Berufsgenossenschaften, St. Augustin

5. Riemann A (2001) Narbenmassage bei Brandverletzten. Vortrag bei Jahrestagung der Deutschsprachigen Arbeitsgemeinschaft für Verbrennungsbehandlung (DAV), 7.-10.1.2001, Obertauern, Österreich

6. Waldner-Nilson B (1997) Ergotherapie in der Handrehabilitation. In: Waldner-Nilson B (Hrsg) Ergotherapie in der Handrehabilitation, Bd 2. Springer, Berlin Heidelberg New York

7. Ziegenthaler H, Reuter BM, Brückner L (2000) Rehabilitation Brandverletzter - differenziert und individuell. Med Orthop Techn 120: 131137 